\title{
Hearing Loss in Malignant Infantile Osteopetrosis: A Case-Based Review
}

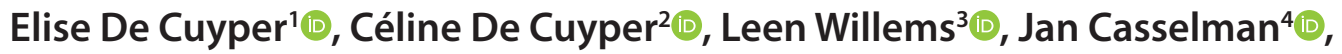 \\ Ingeborg Dhooge ${ }^{1}$ (D), Helen Van Hoecke ${ }^{1}$ (D)
}

'Department of Otorhinolaryngology, Ghent University Hospital, Ghent, Belgium

2Department of Pediatrics, University Hospital Brussels, Jette, Belgium

${ }^{3}$ Department of Internal Medicine and Pediatrics, Ghent University Hospital, Ghent, Belgium

${ }^{4}$ Department of Radiology, AZ St-Jan Brugge AV, Brugge, Belgium

ORCID IDs of the authors: E.D.C. 0000-0002-6371-3476; C.D.C. 0000-0002-0410-8372; L.W. 0000-0003-1230-1049; J.C. 0000-0002-8970-6979; I.D. 0000-0002-5915-1079; H.V.H. 0000-0003-1012-021X.

Cite this article as: De Cuyper E, De Cuyper C, Willems L, Casselman J, Dhooge I, Van Hoecke H. Hearing loss in malignant infantile osteopetrosis: A case-based review. J Int Adv Otol. 2021;17(6):551-558.

Osteopetrosis, or marble bone disease, is a rare genetic disease of bone resorption. It includes a clinically heterogeneous group of conditions that are characterized by increased bone density on radiographs due to a defect in osteoclasts. A most common feature of osteopetrosis of the temporal bone is hearing impairment. This case-based review describes the potential otologic and hearing manifestations of malignant infantile osteopetrosis. The hearing loss can be conductive, sensorineural, late-onset or relapsing. Once the diagnosis is made, referral to an ENT physician for hearing evaluation is indicated. Although otitis media with effusion is the most frequent cause of conductive hearing loss with autosomal recessive osteopetrotic patients, audiometry after tympanostomy tube placement to check for additional causes of hearing loss is highly recommended. As otological manifestations may worsen over time, accurate and regular follow-up by audiometry is necessary. According to our knowledge, this is the first case report in the literature of dehiscent jugular bulb in a patient with osteopetrosis.

KEYWORDS: Dehiscent jugular bulb, hearing loss, malignant infantile osteopetrosis, otologic manifestations

\section{INTRODUCTION}

Osteopetrosis, or marble bone disease, is a rare genetic bone disease initially described by Albers-Schönberg in 1904. Osteopetrosis includes a clinically heterogeneous group of conditions characterized by increased bone density due to a defect in bone reabsorption by osteoclasts. The osteoclastogenesis as well as the osteoclastic activity may be distorted. This results in excessive deposition of immature bone, thickening of the cortical bones, and failure of the outgrowth of spaces in the cranial vault. ${ }^{1-6}$

\section{ETIOLOGY}

Mutations in many different genes have been identified. Osteopetrosis can be inherited in an autosomal dominant (autosomal dominant osteopetrosis (ADO), osteopetrosis tarda, or the adult type), autosomal recessive (autosomal recessive osteopetrosis (ARO) or malignant infantile type), or X-linked recessive pattern. . $^{1,2,5,7-9}$

Concerning ARO, 2 types exist: osteoclast-rich ARO and osteoclast-poor ARO. Multiple mutations (TCIRG1, CLCN7, OSTM1, SNX10, etc.) lead to osteoclast-rich ARO: osteoclasts are abundant but have severely impaired bone resorption function. Due to impaired endosomal and lysosomal vesicle trafficking, osteoclasts cannot actively secrete specialized enzymes to dissolve bone mineral and degrade bone matrix. Mutations in other genes (TNFSF11, TNFRSF11A, RANK, and RANKL) accounting for osteoclast differentiation and activation lead to osteoclast-poor ARO, in which no mature osteoclasts are present. ${ }^{1,25-9}$

Estimating the overall incidence of osteopetrosis is difficult. Previous literature mentions 1 in 250000 births for ARO and 1 in 20000 births for ADO..$^{1-4,6,8}$ 


\section{Clinical Presentation}

Clinical symptoms of osteopetrosis vary greatly in their presentation and severity. The spectrum ranges from neonatal onset with life-threatening complications (due to bone marrow failure) to incidental findings of osteopetrosis on radiographs. The increased bone mass can result in phenotypic features such as macrocephaly and altered craniofacial morphology. In addition, this may paradoxically weaken the bone, resulting in a brittle bone with a predisposition to fractures and osteomyelitis. More importantly, the increased bone mass has an impact on the bone marrow and nervous systems. The expanding bone can narrow nerve foramina causing among others deafness, blindness, facial palsy, swallowing difficulties, and atrophy of the retina. The venous outflow may be compressed too, with raised intracranial pressure and hydrocephalus. Severe dental caries, dental abscesses, or delayed tooth development are also common, as well as growth retardation/failure and delayed psychomotor development. ${ }^{1-3,5,7,8,10}$

$\mathrm{ARO}$, the most severe type, is associated with diminished life expectancy and is fatal if untreated. Symptoms of ARO appear soon after birth and may present with early and late-onset neonatal sepsis. ARO patients are at risk for developing hypocalcemia with possibly tetanic seizures and secondary hyperparathyroidism. The most severe complication is bone marrow suppression. This may result in pancytopenia with secondary infections and/or (central nervous system (CNS)) bleeding, hepatosplenomegaly, and myeloid metaplasia. Hematologic symptoms usually present before neurological complications. ${ }^{1-3,5,7,8}$

This review highlights the middle ear manifestations in malignant infantile osteopetrosis.

\section{Diagnosis}

Diagnosis is based on clinical and radiographic evaluation, confirmed by bone biopsy and genetic testing. Bone biopsy can distinguish between osteoclast-poor and -rich subtypes. ${ }^{1-3,6,11}$

For ARO, early diagnosis and treatment are important in order to detect hematologic abnormalities and possible complications early and to prevent disease progression before irreversible neurological sequelae occur. Most untreated patients will die in the first decade due to secondary infections and/or (CNS) bleeding, as a complication of bone marrow suppression. ${ }^{1-3,6-8,11}$

\section{Therapy}

Osteopetrosis therapy depends on the symptoms and severity of the disease and requires a multidisciplinary team approach. The best therapeutic approach for patients with ARO is allogeneic hematopoietic stem cell transplantation (HSCT). If HSCT is not appropriate, corticosteroids may be considered. Long-term treatment with interferon-gamma can be considered experimental in ARO. Studies are in progress to test gene and cell therapies, small interfering RNA approach, and novel pharmacologic treatments. ${ }^{1,2,5,6,11}$

\section{CASE PRESENTATION}

Written informed consent for the publication of the clinical details and clinical images was obtained from the parents. A copy of the consent form is available for review by the Editor of this journal.
This male patient was born after an uncomplicated pregnancy and was delivered at 38 gestational weeks by cesarean section (because of cephalopelvic incongruence) with a birth weight of $2840 \mathrm{~g}(\mathrm{SD}-2.0)$, height of $48 \mathrm{~cm}$ (SD -1.0), and head circumference of $33.5 \mathrm{~cm}$ (SD -1.0). There was no history of consanguinity or known genetic disorders in the family. At the age of 3 months, he was admitted to the pediatric intensive care unit because of an unfavorable course of RSV (respiratory syncytial virus) infection with failure to thrive and prominent bulging of the anterior fontanel. Lumbar puncture showed severe intracranial hypertension, and CSF (cerebrospinal fluid) analysis was negative for central nervous system infection. Further imaging demonstrated extensive external hydrocephalus with the need for ventriculoperitoneal shunt placement resulting in rapid improvement of the clinical condition. Moreover, a deflection of the growth curve was noticed. Radiographs of the entire skeleton showed generalized sclerosis of the bone, matching with the diagnosis of infantile osteopetrosis.

Bone marrow biopsy confirmed osteoclast-poor osteopetrosis; genetic testing found a homozygous TCIRG1 mutation. As the diagnosis of ARO was made, he underwent allogeneic HSCT at the age of 7 months after conditioning with busulphan, fludarabin, melphalan, and anti-thymocyte globulin.

After the diagnosis of ARO, he was referred to the department of Otorhinolaryngology for clinical and audiological evaluation. He had passed the newborn hearing screening and brainstem audiometric testing at the age of 7 months (just before allogeneic bone marrow transplantation) was also normal. Neurologic and ophthalmologic evaluation revealed normal cranial nerve functions except for a suspected impaired vision of the right eye, which was later confirmed as a severely impaired right-sided vision.

During further regular follow-up at our Otorhinolaryngology Department, recurrent episodes of acute otitis media and otitis media with effusion (OME) have been noted and observed since the age of 3 years. There was no increased frequency of other upper airway infections and were no signs of upper airway obstruction. After a period of watchful waiting, bilateral tympanostomy tubes were scheduled because of persistent OME with impact on hearing acuity (Figure 1, free-field audiometry preoperative). Peroperatively, a bluish mass was seen behind the right tympanic membrane, compatible with a dehiscent jugular bulb. Middle ear effusion was present in both ears. Placement of tympanostomy tubes was proceeded carefully at an anterosuperior position for the right ear. A hearing test postoperatively revealed a low frequency, severe conductive hearing loss for the right ear. The left ear showed normal hearing (Figure 1, pure tone audiometry postoperative).

Cone beam CT scan of the petrous bone showed bilaterally a small tympanic cavity with thickened superstructures and footplates of the stapes. Furthermore, as clinically suspected, a right dehiscent and protruding jugular bulb in contact with the tympanic membrane and malleus handle, occluding the round window niche and probably in contact with the head of the stapes and the incudostapedial joint was detected. On both sides, there was an important upward and 

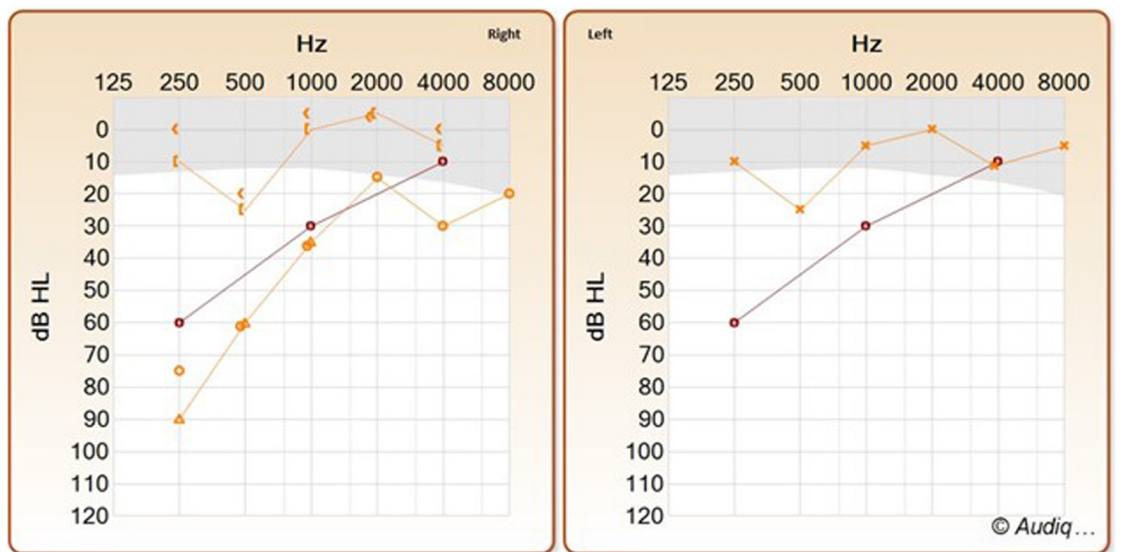

-10 Free-field audiometry preoperative

= Pure tone audiometry postoperative

Figure 1. Free-field audiometry preoperative and pure tone audiometry postoperative for the right and left ear.

medial angulation of the petrous bone apex resulting in a noticeable inclined and verticalized tegmen (Figure 2).

Rehabilitation with a conventional or bone-anchored hearing aid for the right ear was advised. Until now, after 10 years of follow-up, there was no recurrence of otitis media or need for replacing tympanostomy tubes. Audiometric thresholds still remain stable, and good functional results are achieved with a conventional behind-the-ear hearing aid on the right side. Apart from monocular blindness, the patient has a good general health and shows a favorable development with stable blood values since allogeneic HSCT.

\section{Clinical and Research Consequences}

This case represents a young child diagnosed with infantile ARO at the age of 3 months and treated with allogeneic HSCT at the age of 7 months, presenting with recurrent episodes of acute otitis media and otitis media with effusion. Despite the placement of tympanostomy tubes, unilateral conductive hearing loss persisted.
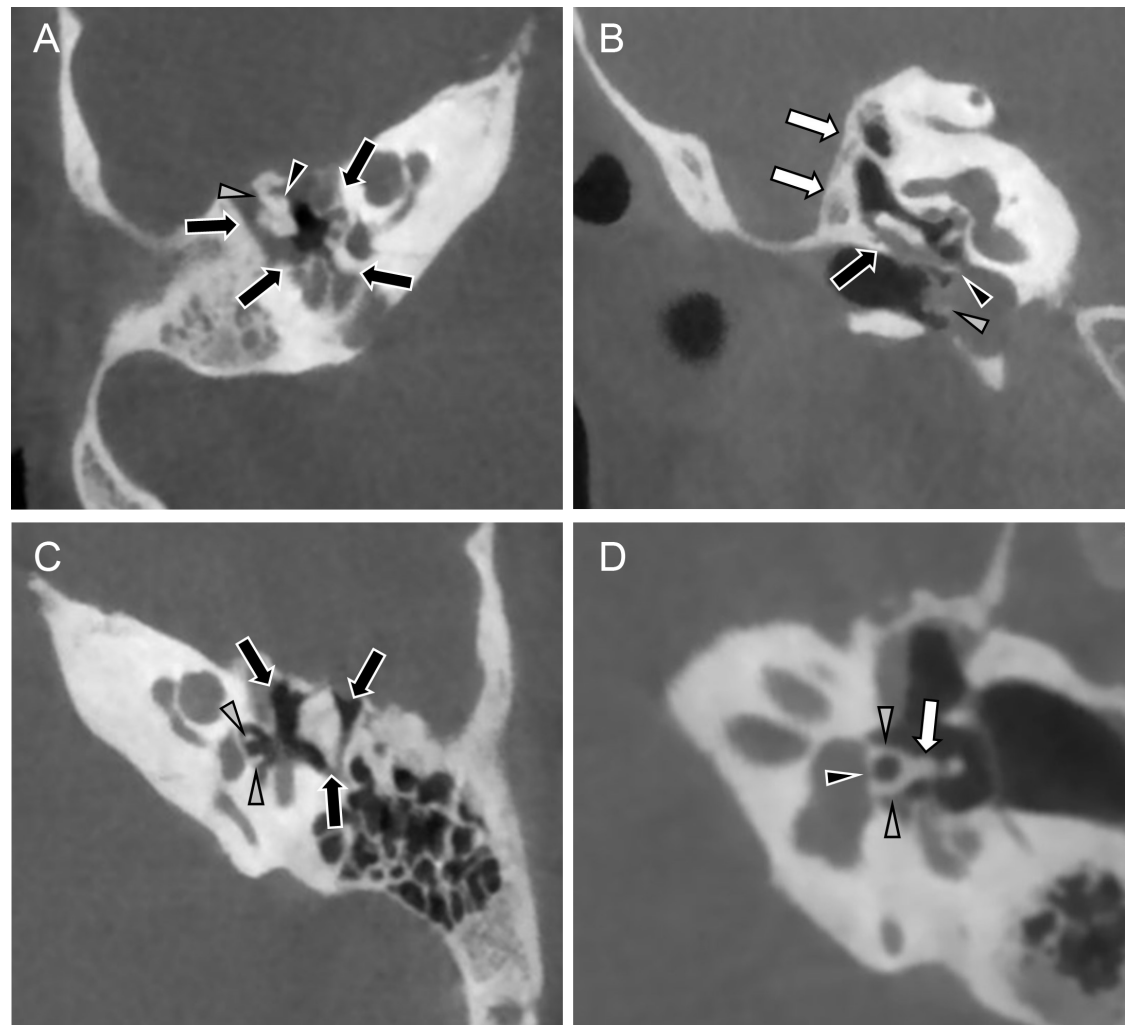

Figure 2. (A) Axial CBCT of the right temporal bone. Small middle ear cavity (arrows), malleus head (black arrowhead) fixed to the anterior tympanic wall by a malleus bar (grey arrowhead). (B) Coronal CBCT of the right temporal bone. Small middle ear cavity (black arrow) with a very steep tegmen (white arrows). A dehiscent protruding jugular bulb makes contact with the tympanic membrane and a grommet (grey arrowhead) and the malleus handle (black arrowhead). (C) Axial CBCT of the left temporal bone. Very small tympanic cavity (arrows) with almost blocked ossicles. Thickened crura of the stapes (arrowheads). (D) Double oblique CBCT images through the left incudostapedial joint. Notice the thickened crura (grey arrowheads), thickened capitulum (arrow), and the thickened footplate (black arrowheads) of the left stapes. 
Hearing loss is the most common feature of osteopetrosis of the temporal bone for patients with ARO. Both conductive and sensorineural hearing loss can be associated. ${ }^{1,4,12-16}$ Conductive hearing loss, estimated to affect $78 \%$ of the patients with $A R O$, is the most frequent. ${ }^{2,16}$ An overview of previous studies describing otological manifestations in osteopetrotic patients is represented in Table 1. As well normal hearing as conductive, sensorineural and mixed hearing loss have been associated with ARO; uni- or bilateral, ranging from mild to severe. Even some cases describe a late-onset hearing loss (both conductive and sensorineural) as being progressive. ${ }^{12,16,17}$ Relapsing hearing loss with tinnitus was attributed to osteopetrosis in 1 study based on clinical and radiographic evaluation of the skeleton. ${ }^{18}$

Different factors contributing to conductive hearing loss in osteopetrotic patients have been suggested. The leading cause is narrowing of the bony portion of the eustachian tube resulting in recurrent episodes of otitis media with effusion. The associated conductive hearing loss is mostly transient and may require (repeated) placement of tympanostomy tubes. ${ }^{14,12,16,17,19}$ Another contributing factor is atresia or narrowing of the external auditory canal. Also, ossicular deformities due to osteoclast dysfunction can reduce the ossicular mobility leading to impaired sound transmission and thus conductive hearing loss. ${ }^{1,4,12,16,17,19}$ A study by Kanzaki et al. ${ }^{13}$ analyzed the impact of affected auditory ossicles for hearing in osteopetrotic mice. Osteoclasts play a significant role in bone resorption, which also includes thinning of the ossicles and enlargement of the tympanic cavity to ensure maximal vibration. As a result of the distorted osteoclastic activity, thicker auditory ossicles and smaller volume of the tympanic cavity are seen in osteopetrotic mice compared with control mice. ${ }^{13}$ Actually, a thicker and fixated stapes has previously been reported in osteopetrosis to cause conductive hearing loss. ${ }^{1,15-17,19}$ Furthermore, the conductive hearing loss in osteopetrotic patients may be due to a thickened fibrous layer of the tympanic membrane. This may also explain the persistent type $B$ on tympanometry during follow-up., 12,16,17 In addition, also sensorineural hearing loss can be found in patients with ARO. Defective bone development and excessive bone formation in the internal auditory canal can result in auditory nerve compression causing sensorineural hearing loss.,12,19 Another plausible explanation for the sensorineural hearing loss is excessive bone formation compressing cochlear blood supply. ${ }^{4,16,19}$ An overview of the different mechanisms leading to hearing loss in osteopetrosis is represented in Table 2 . As some cases describe a progressive late-onset hearing loss (both conductive and sensorineural), this may suggest that the aforementioned otological manifestations may worsen with time, resulting in decreased hearing performance. ${ }^{4,20}$ Even a successful allogeneic HSCT may neither prevent nor improve (progressive) middle ear changes due to osteopetrosis. Hearing loss has also been reported in ADO but is rather rare. ${ }^{2,20}$ Cranial nerve compression leading to hearing loss would affect around $5 \%$ of ADO individuals. ${ }^{2}$ Vestibular symptoms as a part of osteopetrosis have rarely been described but are insufficiently investigated. ${ }^{1}$

Concerning our patient with a right-sided conductive hearing loss, similar ossicular deformities as described earlier were found on CT scan: small tympanic cavity and thickened superstructures and footplates in both ears (Figure 2). However, as our patient did not demonstrate hearing loss at the left side, we assume that the ossicular changes did not lead to ossicular fixation and clinically apparent hearing loss.
A possible explanation for the unilateral conductive hearing loss in our patient is a dehiscent jugular bulb. The jugular bulb is the transition point from sigmoid sinus to internal jugular vein. It is usually located below the floor of hypotympanum and separated from the middle ear by a layer of bone. ${ }^{21-27}$ In $0.5 \%-1.7 \%$, this bony septum is absent which is defined as a dehiscent jugular bulb. ${ }^{23,24}$ Consequently, the jugular bulb can expand and protrude into the middle ear, which can be noticed during otoscopy as a red-purplish mass in the posterior-inferior quadrant of the tympanic membrane. Both conductive hearing loss and pulsatile tinnitus are associated with a dehiscent jugular bulb. 22-25,27-29 Impinging of the dehiscent jugular bulb on the tympanic membrane, middle ear ossicles, or round window can cause conductive hearing loss. ${ }^{24,26,27}$ Furthermore, this important anatomic variation can entail significant morbidity and even mortality during middle ear surgery. Several cases of jugular injury and massive bleeding, in particular during myringotomy and tympanomeatal flap elevation, have been reported in the past. ${ }^{25,27,28}$

In current literature, the etiology of jugular bulb anomalies is poorly understood. Moreover, there is no evidence of a dehiscent jugular bulb associated with osteopetrosis. Some studies suppose that mastoid pneumatization could play an important role in jugular bulb development after birth. ${ }^{28,30}$ A tendency for the development of a high jugular bulb in poorly pneumatized mastoids has been suggested in 1977. ${ }^{31}$ One theory proposes that the distance from the sigmoid sinus to the external auditory canal is shorter in cases of little/absent pneumatization. ${ }^{30}$ As poor pneumatization of the mastoid bone has been associated with osteopetrosis before, this could explain the location of the prominent dehiscent jugular bulb seen in our patient although more recent research found no correlation between the pneumatization of the temporal bone and the position of the jugular bulb. 1,14,16,17,19,31 Secondly, it has been suggested that abnormal venous flow is a contributing factor for jugular bulb anomalies. ${ }^{30}$ As described earlier, osteopetrosis can result in a compressed venous outflow and increased intracranial pressure due to increased bone mass. However, the reason why this patient developed a unilateral dehiscent jugular bulb - and not bilaterally - could not be revealed. Lastly, Moore ${ }^{32}$ related a dehiscent jugular bulb to abnormal bone formation and/or bone remodeling, for example, as seen in Paget's disease. ${ }^{32}$ The contribution of abnormal bone remodeling in osteopetrotic patients to the development of jugular bulb anomalies is still unclear today, and further investigation is warranted.

In summary, it is mandatory to evaluate each osteopetrotic patient for hearing loss. The associated hearing loss is mostly of a conductive and transient nature due to $\mathrm{OME}$, but it is recommended to re-evaluate hearing after tympanostomy tube placement as osteopetrosis may have other causes of conductive hearing loss. Our patient was treated with HSCT at the age of 7 months although recurrent middle ear problems and a dehiscent jugular bulb were only established around the age of 3 years. Therefore, it is plausible that, even after successful treatment, children may develop otological deformities. Moreover, as late-onset and progressive hearing loss have been described in ARO patients, early and subsequent otological and audiological follow-up is strongly recommended thus referral to an otorhinolaryngologist is highly advised. The maximum age of lateonset hearing loss is currently unknown; more research is warranted to determine the duration of follow-up. 


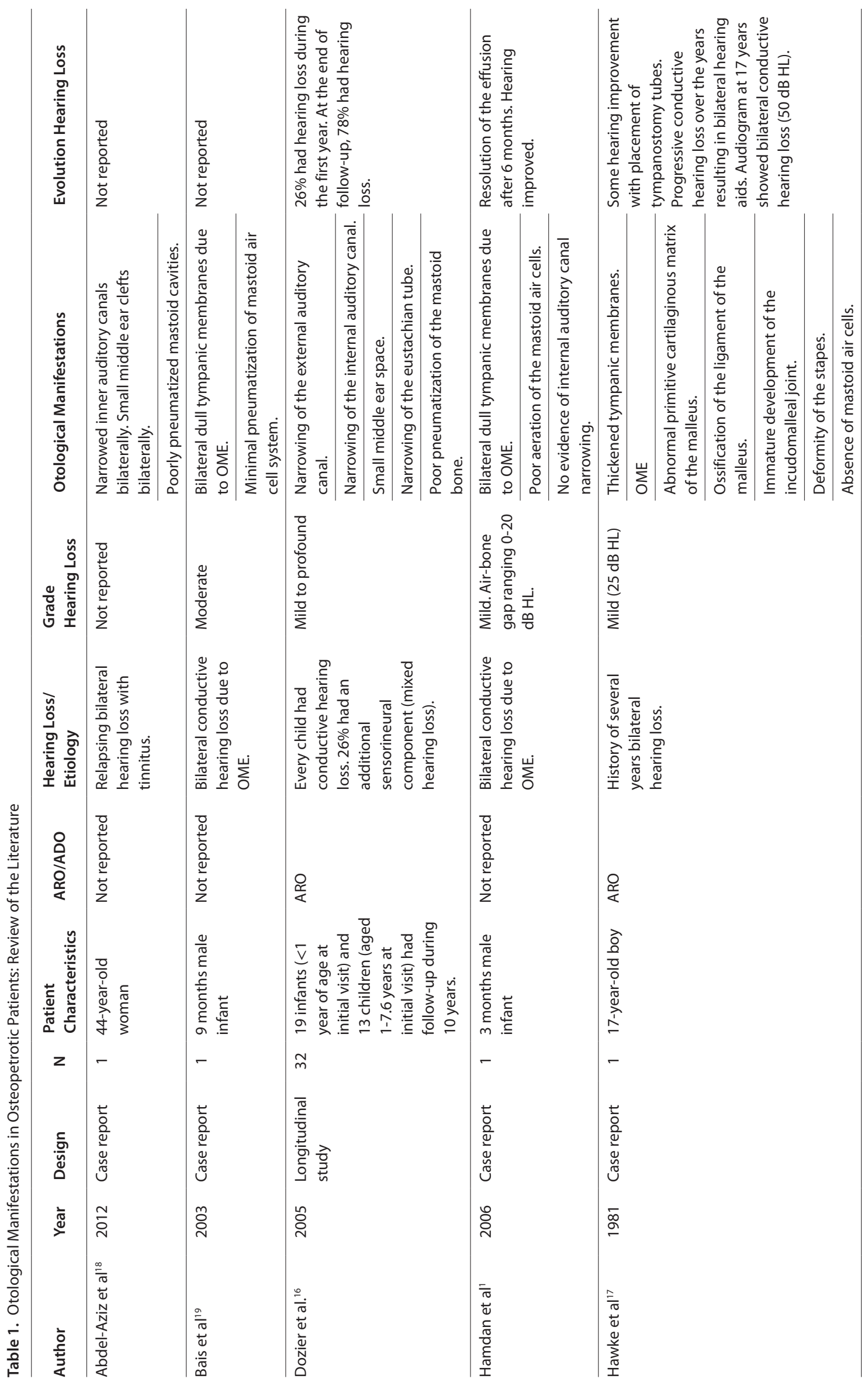




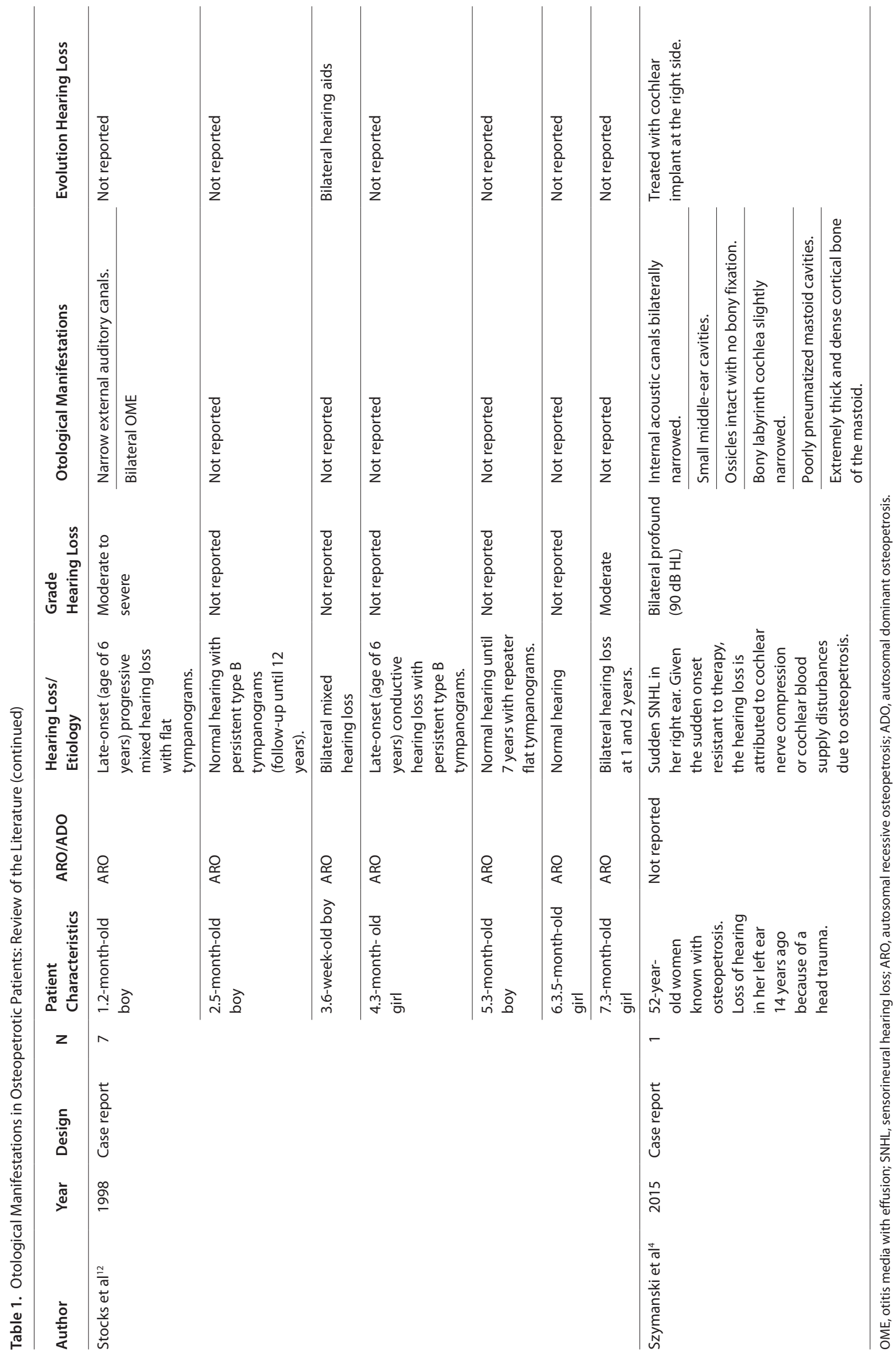


Table 2. Described Otological Manifestations in Malignant Infantile Osteopetrosis. ${ }^{1,4,12,19}$

\begin{tabular}{ll}
\hline Otological Manifestations \\
\hline Conductive \\
hearing loss & Calcified, sclerotic, or fixed ossicles. \\
\cline { 2 - 2 } & Deformity of the stapes. \\
\cline { 2 - 2 } & Exostosis in the middle ear/small middle-ear cavities. \\
\cline { 2 - 2 } & Narrowed eustachian tube. \\
\cline { 2 - 2 } $\begin{array}{l}\text { Otitis media with effusion/recurrent otitis media. } \\
\text { hensorineural }\end{array}$ & Partial bony atresia of the external auditory meatus. \\
\cline { 2 - 2 } & Cochlear blood supply disturbances. \\
\cline { 2 - 2 } & Cochlear nerve compression. \\
\hline Mastoid & Poor pneuming of the internal auditory canal. \\
\hline
\end{tabular}

\section{CONCLUSIONS}

This case-based review was performed to inform about the need for rapid diagnosis as ARO may entail serious morbidities and even cause mortality. Osteopetrosis should be kept in mind as a rare cause of primarily skeletal manifestations such as fractures and osteomyelitis in late childhood or adolescence. The presence of primary neurodegeneration, mental retardation, hematological symptoms, or immune system involvement may point to rarer osteopetrosis variants.

Furthermore, this case report emphasizes the otological manifestations of malignant infantile osteopetrosis. The hearing loss can be conductive, sensorineural, late-onset, or relapsing. Once the diagnosis is made, referral to an ENT physician for hearing evaluation is indicated. Although OME is the most frequent cause of conductive hearing loss in ARO patients, audiometry after tympanostomy tube placement to check for additional causes is highly advised. As otological manifestations may worsen with time, accurate and regular follow-up by audiometry is necessary. According to our knowledge, this is the first case report in the literature of dehiscent jugular bulb in a patient with osteopetrosis.

Ethics Committee Approval: Approval of the Commission for Medical Ethics was obtained (EC/096-2020/sds).

Informed Consent: Written informed consent for the publication of the clinical details and clinical images was obtained from the parents. A copy of the consent form is available for review by the Editor of this journal.

Peer-review: Externally peer-reviewed.

Author Contributions: Concept - L.W., J.C., H.V.H.; Design - L.W., J.C., H.V.H.; Supervision - L.W., J.C., H.V.H., I.D.; Resource - none; Materials - E.D.C., C.D.C., J.C., H.V.H.; Data Collection and/or Processing - E.D.C., C.D.C., J.C., H.V.H.; Analysis and/or Interpretation - E.D.C., C.D.C., J.C., I.D., H.V.H.; Literature Search E.D.C., C.D.C., H.V.H.; Writing - E.D.C, C.D.C, J.C., H.V.H.; Critical Reviews - E.D.C., C.D.C., L.W., J.C., I.D., H.V.H.

Acknowledgements: Gratitude to the parents and the patient.
Conflict of Interest: The authors have no conflict of interest to declare.

Financial Disclosure: The authors declared that this study has received no financial support.

\section{REFERENCES}

1. Hamdan AL, Nabulsi MM, Farhat FT, Haidar RK, Fuleihan NS. When bone becomes marble: head and neck manifestations of osteopetrosis. Paediatr Child Health. 2006;11(1):37-40. [CrossRef]

2. Stark Z, Savarirayan R. Osteopetrosis. Orphanet J Rare Dis. 2009;4(1):5. [CrossRef]

3. National Organization for Rare Disorders (NORD). Osteopetrosis. Rare Disease Database. 2018. Available at: https://rarediseases.org/rare-disea ses/osteopetrosis/.

4. Szymanski M, Zasławska K, Trojanowska A, Szymanska A, Zadrozniak M. Osteopetrosis of the temporal bone treated with cochlear implant. J Int Adv Otol. 2015;11(2):173-175. [CrossRef]

5. Teti A, Econs MJ. Osteopetroses, emphasizing potential approaches to treatment. Bone. 2017;102:50-59. [CrossRef]

6. Bubshait DK, Himdy ZE, Fadaaq O, Alshmas HI. Malignant infantile osteopetrosis: a case report. Cureus. 2020;12(1):e6725. [CrossRef]

7. Sobacchi C, Schulz A, Coxon FP, Villa A, Helfrich MH. Osteopetrosis: genetics, treatment and new insights into osteoclast function. Nat Rev Endocrinol. 2013;9(9):522-536. [CrossRef]

8. Gillani S, Abbas Z. Malignant infantile osteopetrosis. J Ayub Med Coll Abbottabad. 2017;29(2):350-352.

9. Villa A, Guerrini MM, Cassani B, Pangrazio A, Sobacchi C. Infantile malignant, autosomal recessive osteopetrosis: the rich and the poor. Calcif Tissue Int. 2009;84(1):1-12. [CrossRef]

10. Dowlati D, Winston KR, Ketch LL, et al. Expansion cranioplasty with jackscrew distracters for craniosynostosis and intracranial hypertension in transplanted osteopetrosis. Pediatr Neurosurg. 2007;43(2):102-106. [CrossRef]

11. Wu CC, Econs MJ, DiMeglio LA, et al. Diagnosis and management of osteopetrosis: consensus guidelines from the osteopetrosis working group. J Clin Endocrinol Metab. 2017;102(9):3111-3123. [CrossRef]

12. Stocks RMS, Wang WC, Thompson JW, Stocks MC, II, Horwitz EM. Malignant infantile osteopetrosis: otolaryngological complications and management. Arch Otolaryngol Head Neck Surg. 1998;124(6):689-694. [CrossRef]

13. Kanzaki S, Takada Y, Niida S, et al. Impaired vibration of auditory ossicles in osteopetrotic mice. Am J Pathol. 2011;178(3):1270-1278. [CrossRef]

14. Shah LM, Wiggins RH, 3rd. Imaging of hearing loss. Neuroimaging Clin $N$ Am. 2009;19(3):287-306. [CrossRef]

15. Aharinejad S, Grossschmidt K, Franz P, et al. Auditory ossicle abnormalities and hearing loss in the toothless (osteopetrotic) mutation in the rat and their improvement after treatment with colony-stimulating factor-1. J Bone Miner Res. 1999;14(3):415-423. [CrossRef]

16. Dozier TS, Duncan IM, Klein AJ, Lambert PR, Key LL, Jr. Otologic manifestations of malignant osteopetrosis. Otol Neurotol. 2005;26(4):762-766. [CrossRef]

17. Hawke M, Jahn AF, Bailey D. Osteopetrosis of the temporal bone. Arch Otolaryngol. 1981;107(5):278-282. [CrossRef]

18. Abdel-Aziz T, Upile T, Scholtz LU, Sudhoff HH. Osteopetrosis of the temporal bone. Otol Neurotol. 2012;33(6):e45-e46. [CrossRef]

19. Andreu-Arasa VC, Sung EK, Fujita A, Saito N, Sakai O. Otosclerosis and dysplasias of the temporal bone. Neuroimaging Clin N Am. 2019;29(1):2947. [CrossRef]

20. Bais AS, Sardana P, Arora M. Osteopetrosis-variable otorhinolaryngological manifestations. Indian J Otolaryngol Head Neck Surg. 2003;55(2):113116. [CrossRef] 
21. DeHart AN, Shaia WT, Coelho DH. Hydroxyapatite cement resurfacing the dehiscent jugular bulb: novel treatment for pulsatile tinnitus. Laryngoscope. 2018;128(5):1186-1190. [CrossRef]

22. Barr JG, Singh PK. A rare cause of conductive hearing loss: high lateralized jugular bulb with bony dehiscence. Ear Nose Throat J. 2016;95(6):227229. [CrossRef]

23. El-Begermy MA, Rabie AN. A novel surgical technique for management of tinnitus due to high dehiscent jugular bulb. Otolaryngol Head Neck Surg. 2010;142(4):576-581. [CrossRef]

24. Shaikh MF, Mahboubi H, German M, Djalilian HR. A novel approach for surgical repair of dehiscent high jugular bulb. Laryngoscope. 2013;123(7):1803-1805. [CrossRef]

25. Huang BY, Castillo M, Mukherji SK. Temporal bone disorders in children. In: Rossi A, ed. Pediatric Neuroradiology. Berlin, Heidelberg: Springer; 2015:1-113.

26. Toman J, Wu X, Malhotra A, Michaelides E. Conductive hearing loss and the jugular bulb. Clin Neuroradiol. 2016;26(2):235-238. [CrossRef]
27. Koo YH, Lee JY, Lee JD, Hong HS. Dehiscent high-riding jugular bulb presenting as conductive hearing loss: a case report. Med. 2018;97(26): e11067. [CrossRef]

28. Atmaca S, Elmali M, Kucuk H. High and dehiscent jugular bulb: clear and present danger during middle ear surgery. Surg Radiol Anat. 2014;36(4):369-374. [CrossRef]

29. Sayit AT, Gunbey HP, Fethallah B, Gunbey E, Karabulut E. Radiological and audiometric evaluation of high jugular bulb and dehiscent high jugular bulb. J Laryngol Otol. 2016;130(11):1059-1063. [CrossRef]

30. Manjila S, Bazil T, Kay M, Udayasankar UK, Semaan M. Jugular bulb and skull base pathologies: proposal for a novel classification system for jugular bulb positions and microsurgical implications. Neurosurg Focus. 2018;45(1):E5. [CrossRef]

31. Wang CH, Shi ZP, Liu DW, Wang HW, Huang BR, Chen HC. High computed tomographic correlations between carotid canal dehiscence and high jugular bulb in the middle ear. Audiol Neurootol. 2011;16(2):106-112. [CrossRef]

32. Moore PJ. The high jugular bulb in ear surgery: three case reports and a review of the literature. J Laryngol Otol. 1994;108(9):772-775. [CrossRef] 